\title{
Entre Église et théâtre, la fugue de deux musiciennes vénitiennes en 1783
}

\section{Caroline GIRON-PANEL}

\section{(2) OpenEdition}

1 Journals

\section{Édition électronique}

URL : https://journals.openedition.org/clio/2752

DOI : $10.4000 /$ clio.2752

ISSN : 1777-5299

Éditeur

Belin

Édition imprimée

Date de publication : 1 avril 2007

Pagination : 93-113

ISBN : 978-2-85816-900-9

ISSN : 1252-7017

Référence électronique

Caroline GIRON-PANEL, " Entre Église et théâtre, la fugue de deux musiciennes vénitiennes en 1783 », Clio. Histoire, femmes et sociétés [En ligne], 25 | 2007, mis en ligne le 01 juin 2009, consulté le 22 avril 2022. URL : http://journals.openedition.org/clio/2752 ; DOI : https://doi.org/10.4000/clio.2752

Ce document a été généré automatiquement le 22 avril 2022.

Tous droits réservés 


\title{
Entre Église et théâtre, la fugue de deux musiciennes vénitiennes en
} 1783

\author{
Caroline GIRON-PANEL
}

1 Les archives vénitiennes regorgent d'histoires d'amours contrariées, de fugues en gondole et autres mariages secrets, qui sont pour les romanciers des sources intarissables d'inspiration. Mais il arrive parfois que l'historien puisse lui aussi faire son miel de ces documents, lorsque l'anecdote vient éclairer un aspect méconnu de l'histoire sociale.

2 Le fonds Provveditori sopra Ospedali e Luoghi pii (Provéditeurs aux hôpitaux et aux pieux établissements), conservé à l'Archivio di Stato de Venise, renferme ainsi un dossier intitulé "Ferrarese e Sacchetti fuggite ${ }^{1}$ ", qui contient (comme son nom l'indique) toute une série de documents relatifs à la fugue de deux jeunes femmes, Bianca Sacchetti et Andrianna Ferrarese. Musiciennes à l'ospedale des Mendicanti, elles s'étaient enfuies en gondole, dans la nuit du 8 au 9 janvier 1783, en compagnie de Luigi dal Bene, fils d'Agostino dal Bene, consul de Rome à Venise. Retrouvées le lendemain dans une auberge de Portogruaro, sur la Terre Ferme, elles avaient été ramenées sous la contrainte à l'ospedale, tandis que Luigi dal Bene prenait la fuite. Andrianna Ferrarese ayant perdu sa virginité dans l'aventure, elle fut expulsée de l'ospedale, tandis que Bianca Sacchetti, jeune et influençable, fit amende honorable et put être réintégrée dans l'institution, où elle passa le restant de ses jours.

3 L'histoire pourrait sembler anecdotique s'il ne s'agissait pas d'un témoignage unique sur deux des plus célèbres musiciennes italiennes de la fin du XVIII ${ }^{e}$ siècle. Au-delà de l'aspect romanesque, cette série de documents nous donne en outre, par des voies détournées, de nombreuses informations sur la vie quotidienne des «filles du chœur » d'un ospedale vénitien, sur leurs sentiments à l'égard de cette institution et sur leurs aspirations à une vie meilleure. Le dossier contient seize documents de nature très diverse. On trouve ainsi, pêle-mêle, les lettres des présidents de l'ospedale aux différentes autorités de Venise, les mots d'amour que s'échangeaient Andrianna 
Ferrarese et Luigi dal Bene, ou encore le rapport de la sage-femme chargée d'examiner les jeunes femmes afin de déterminer si elles méritaient encore le nom de putte ${ }^{2}$. La variété de ces documents offre différents éclairages sur une affaire complexe, dont on peut percevoir, aujourd'hui encore, l'implication pour les jeunes musiciennes et leur institution.

Le décor : les ospedali de Venise

4 Les quatre ospedali de Venise avaient été créés, au Moyen-Âge ou au début du XVI ${ }^{e ̀}$ siècle, pour venir en aide à différentes catégories de nécessiteux. L'ospedale de San Lazzaro, le plus ancien, était destiné à l'origine à accueillir les lépreux, puis avait étendu son champ d'action aux mendiants et aux vieillards (d'où son nom, à partir de 1600, de "San Lazzaro e dei Mendicanti»). Celui de la Pietà, créé au XIVè siècle par le franciscain Pierre d'Assise, accueillait les enfants abandonnés. L'ospedale des Incurabili avait pour vocation de soigner les malades incurables, notamment les syphilitiques, tandis que celui des Derelitti (ou «Abandonnés », également appelé "Ospedaletto » au XVIII ̀̀ siècle), recueillait les pauvres menacés par la famine. À l'exception de la Pietà, exclusivement destinée à l'accueil des enfants "exposés", tous les ospedali de Venise recueillaient également les orphelins, à condition que ceux-ci fussent Vénitiens, baptisés et pourvus d'une attestation de pauvreté.

5 Ces institutions avaient pour but de venir en aide aux laissés-pour-compte de la République, mais également de leur donner les moyens de leur salut. Illustrant ce que Brian Pullan a appelé la "nouvelle philanthropie ", elles fournissaient une instruction de base à tous les enfants qu'elles recueillaient et leur apprenaient un métier, afin qu'ils ne fussent pas réduits à la mendicité pour survivre. L'enseignement de la musique n'était qu'une facette de cette instruction que recevaient les orphelins. À l'origine, les enfants n'apprenaient que les rudiments du chant, afin d'être capables d'accompagner les offices religieux qui étaient dits plusieurs fois par jour dans la chapelle de chaque ospedale. Les quatre institutions avaient alors un rôle très secondaire dans la vie musicale vénitienne. D'autres lieux étaient par essence dédiés à la musique sacrée : certaines églises paroissiales ou conventuelles (notamment Santa Maria Gloriosa dei Frari ou Santi Giovanni e Paolo) mettaient un point d'honneur à employer d'excellents organistes et les plus fortunées employaient parfois des chanteurs de Saint-Marc à l'occasion des grandes fêtes religieuses. La basilique ducale resta en effet au cœur de la musique sacrée vénitienne jusqu'au XVIII ${ }^{e ̀ ~ s i e ̀ c l e, ~ m e ̂ m e ~ s i ~ d ' a u t r e s ~ p o ̂ l e s ~ e ́ m e r g e ̀ r e n t ~ a ̀ ~}$ partir du début du siècle précédent. La chapelle musicale de Saint-Marc était dirigée par un maître de chapelle, poste qu'occupèrent à la fin de leur carrière plusieurs " maîtres de chœur » des ospedali. Les chœurs étaient composés de chanteurs adultes, engagés pour trois ou quatre ans et parfois recrutés hors de Venise, notamment les castrats (souvent formés dans les conservatoires napolitains). La schola cantorum fondée dès 1403 pour enseigner le chant aux petits garçons ne fournissait qu'un petit nombre de chanteurs adultes, seuls les plus doués ayant l'honneur de chanter à la chapelle ducale. Les autres pouvaient éventuellement être employés ponctuellement par des églises secondaires de Venise, donner des leçons particulières ou bien tomber dans la misère s'ils avaient perdu leur voix et ne connaissaient aucun autre métier que celui de chanteur.

$6 \quad$ Le contexte dans lequel la musique se développa dans les ospedali n'était donc pas des plus favorables. Les archives nous apprennent d'ailleurs que les gouverneurs hésitèrent longuement avant d'accepter les dépenses nécessaires à la création d'un chœur digne 
de ce nom. Outre l'aspect financier, essentiel pour ces institutions vivant essentiellement de la charité publique, la musique apparaissait comme une activité suspecte, qu'il convenait de surveiller afin que la louange ne se muât pas en divertissement. Il n'était pas question de dilapider en une activité futile l'argent destiné aux pauvres, et l'interdiction répétée de jouer d'autres instruments que l'orgue allait en ce sens: la musique sacrée avait pour unique objet la prière, non le plaisir des auditeurs. Malgré leurs hésitations initiales, les gouverneurs des ospedali réalisèrent dès les premières années du XVIİ̀ siècle que l'activité musicale pouvait être une source de revenus importante. En effet, les chœurs des quatre institutions virent le jour au moment même où l'opéra triomphait dans les théâtres de Venise... fermés plusieurs mois par an. Les mélomanes se pressaient alors nombreux dans les chapelles de la Pietà, des Mendicanti, des Derelitti ou des Incurabili pour entendre les voix si pures de ces putte, comme les appelaient affectueusement les Vénitiens, invisibles derrière leurs hautes grilles. Simples offices chantés à l'origine, les cérémonies religieuses des ospedali se transformèrent progressivement en de véritables concerts de musique sacrée, avec exécution d'oratorios, éditions de livrets et location (payante) de chaises. La qualité des musiciennes et la renommée des musiciens chargés de composer des œuvres sans cesse nouvelles attiraient des auditeurs toujours plus nombreux, qui ne manquaient pas d'exprimer leur contentement en remplissant généreusement les troncs laissés à leur disposition dans l'église.

7 Afin d'attirer le public, les gouverneurs des ospedali devaient veiller à ce que la musique fût toujours d'excellente qualité. Les influences de la musique profane se firent progressivement sentir, notamment lorsqu'au XVIII è siècle plusieurs «maitres de chœur " poursuivirent en parallèle une carrière au théâtre. Baldassare Galuppi, par exemple, dirigeait le chœur des Mendicanti tout en mettant en musique plusieurs opéras comiques de Carlo Goldoni ${ }^{4}$. Afin d'assurer un excellent niveau d'enseignement, les gouverneurs engagèrent les meilleurs musiciens de Venise, puis d'Italie, pour enseigner le chant et les instruments aux petites musiciennes. C'est ainsi qu'Antonio Vivaldi fut professeur de violon à la Pietà à diverses reprises, entre 1703 et 1738.

8 L'une des originalités des ospedali de Venise réside dans le fait que l'enseignement de la musique y ait été réservé aux petites filles. Les petits garçons apprenaient parfois les rudiments du solfège, afin de pouvoir accompagner les offices des religieux hébergés dans les ospedali (où ils enseignaient le catéchisme). Une chapelle privée était mise à leur disposition afin qu'ils puissent réciter l'office des heures, mais il aurait été inconvenant que des jeunes filles viennent y chanter. L'accompagnement musical des offices était donc confié à des orphelins sélectionnés pour leur voix, mais l'instruction musicale qu'ils recevaient ne ressemblait en rien à celle dispensée aux «filles du chœur ». L'exclusion des petits garçons des chœurs des ospedali s'explique par le fait que l'évolution de leur voix était trop incertaine. Les institutions vénitiennes n'ayant pas, à l'inverse des conservatoires de Naples, vocation à former des castrats, les gouverneurs choisissaient uniquement des jeunes filles talentueuses, dont la voix promettait de rester belle à l'âge adulte.

9 À l'origine, les musiciennes étaient choisies parmi les orphelines qui présentaient le plus de dispositions naturelles pour la musique. Les critères d'admission étaient très stricts: outre des critères d'âge, variables selon les institutions et les époques, l'orpheline devait impérativement être de nationalité vénitienne et pouvoir fournir une attestation de baptême, de pauvreté et de «bonnes mœurs », délivrées par le prêtre de 
la paroisse. Au XVIII ${ }^{e}$ siècle, les musiciennes commencèrent à être recrutées sur audition, les conditions d'âge, de pauvreté ou de nationalité n'apparaissant plus comme essentielles pour peu que la jeune fille fût réellement talentueuse... Cette évolution donna naissance à de véritables écoles de musique, qui acceptaient des élèves externes, payant pour recevoir les leçons de musique. Le recrutement sur audition permit aux ospedali d'offrir tout au long du XVIII ${ }^{e}$ siècle des concerts de haut niveau, qui attirèrent les mélomanes de toute l'Europe et contribuèrent à faire de ces institutions typiquement vénitiennes des modèles pour les conservatoires européens.

Dramatis personnae

Le cas d'Andrianna Ferrarese et de Bianca Sacchetti illustre une autre facette de l'histoire musicale des ospedali vénitiens. "Filles du chœur » à l'ospedale des Mendicanti, elles y étaient entrées comme "pauvres" avant d'être choisies pour recevoir un enseignement musical. Les archives nous apprennent que Bianca Sacchetti, née le 22 février 1769 , avait sollicité, par le biais du prêtre de sa paroisse, son admission aux Mendicanti en septembre 1774 car son père l'avait abandonnée et sa mère était impotente. Bien qu'il y eût une place vacante parmi les orphelines, sa supplique fut rejetée car elle n'obtint pas les cinq sixièmes des voix ${ }^{5}$. La demande réitérée en février 1775 fut cette fois acceptée. Il est probable qu'elle ait eu déjà des connaissances en musique, ou du moins qu'elle les ait acquises très rapidement au sein de l'ospedale : dès décembre 1779, elle faisait partie du chœur, et elle apparait trois ans plus tard comme soliste dans le Abraham sacrificium de Francesco Bianchi ${ }^{6}$.

11 Les documents sont moins riches sur les débuts d'Andrianna Ferrarese à l'ospedale des Mendicanti. On sait néanmoins qu'elle fut acceptée dans le chœur en mars 1779, alors qu'elle faisait déjà partie des pensionnaires de l'institution. Elle avait alors vingt-quatre ans, ce qui semble un peu tard pour entrer dans le chœur. En réalité, cette date correspond à sa nomination comme soliste. En effet, le cursus habituel des musiciennes des Mendicanti comportait trois étapes : jusqu'à 16 ans, elles étaient dites incipienti et apprenaient les bases du chant et d'un instrument; entre 16 et 21 ans, elles se perfectionnaient dans la classe des profitienti et jouaient ou chantaient dans le chœur, puis les plus douées d'entre elles accédaient au statut de soliste et d'enseignante (maestra). La date de 1779 marque probablement cette promotion du statut de profitiente à celui de maestra. Cette promotion s'accompagna d'une amélioration des conditions de vie de la jeune femme: vêtue de l'uniforme spécifique des filles du chœur, elle secondait le maitre de chœur et touchait à ce titre seize sous par jour (alors que les ouvrières, aussi appelées "filles du commun" en touchaient quatorze), elle vivait à l'étage avec les autres musiciennes, possédait une chambre à part et était mieux nourrie que les ouvrières. Son nouveau statut de soliste (ou essercitante) apparait clairement dès 1780, avec la mention d'Andrianna Ferrarese dans le rôle d'Achior, sur le livret du De Morte Sisarae de Jacob Avanzini ${ }^{7}$. À partir de cette date, tous les livrets d'œuvres composées pour les Mendicanti sur lesquels figure le nom des interprètes portent son nom. On sait en outre que dès 1770 , elle était considérée comme une excellente musicienne. Charles Burney, qui l'entendit lors de son passage à Venise, écrit à son sujet ${ }^{8}$ :

L'une d'entre elles [les orphelines], la Ferrarese, chanta très bien. Elle avait une étendue de voix extraordinaire et était capable d'atteindre le mi le plus aigu de nos clavecins et de le tenir un temps considérable, avec une belle voix naturelle.

12 En 1783, Bianca Sacchetti et Andrianna Ferrarese étaient donc des musiciennes accomplies, reconnues comme telles et promises à un brillant avenir au sein de leur 
ospedale. Pourquoi avoir choisi de quitter une institution qui les avait charitablement recueillies et leur avait permis de développer leur talent pour la musique?

L'art de la fugue

La fugue semble avoir été organisée par Andrianna Ferrarese et Luigi dal Bene, fils du consul de Rome à Venise. L'une des lettres écrites par Andrianna Ferrarese à sa bienfaitrice, la procuratrice Giovanelli, nous raconte toute l'histoire. Bianca Sachetti et la Ferrarese étaient allées à plusieurs reprises, pendant un an et demi, jouer de la musique et chanter chez le consul de Rome. Celui-ci les avait convaincues qu'elles perdaient leur temps à l'ospedale et que leur talent s'épanouirait bien mieux sur les scènes de théâtre que dans la chapelle des Mendicanti. Il alla jusqu'à dire à Andrianna Ferrarese que s'il avait vingt ans de moins, et s'il n'était pas déjà marié, il l'épouserait et ferait sa fortune. Il proposa finalement de les aider à fuir, de leur fournir de l'argent et des lettres de recommandation.

Andrianna Ferrarese précisait qu'elle s'était enfuie parce que le consul de Rome avait découvert qu'elle aimait son fils, Luigi, et l'avait menacée de faire enfermer celui-ci afin de le châtier. Agostino dal Bene refusait catégoriquement que la chanteuse épousât son fils et avait menacé d'éloigner définitivement les deux amants. Ce sont ces menaces qui incitèrent la Ferrarese et Luigi dal Bene à organiser la fugue. Ce dernier promit le mariage à la musicienne et organisa sa fugue, avec Bianca Sacchetti, de nuit, en gondole. L'un des documents établit l'inventaire de ce qu'Andrianna Ferrarese emporta dans sa fuite: une robe de velours, deux bustiers, une paire de chaussures, une ceinture, six paires de bas, trois chemises, une cape et plusieurs partitions de différents compositeurs.

Ce qui s'est passé dans la gondole et à l'auberge de Portogruaro, ce sont les lettres des présidents de l'ospedale et le rapport de la sage-femme qui nous l'apprennent. La sagefemme examina les deux jeunes femmes à leur retour de Portogruaro afin de déterminer si elles avaient, ou non, conservé leur virginité, condition sine qua non pour demeurer dans l'ospedale. Au sujet d'Andrianna Ferrarese, la sage-femme écrivit que «l'inclination de la Ferrarese envers Del Bene, et les protestations qu'elle fait de l'honnêteté du but poursuivi tendent à laisser penser que les dommages auxquels son corps avait pu être exposé se limitaient à ce qui avait pu se passer entre elle et son ami. De fait, elle dit ingénument s'être prêtée à l'acte qu'elle appelle conjugal, qu'elle a même accompli avec quelque impatience dans la barque, durant le voyage aller, sans se laisser déconcerter par les témoins $»^{9}$.

16 La perte de sa virginité contraint Andrianna Ferrarese à quitter l'ospedale, tandis que Bianca Sacchetti obtient l'autorisation de réintégrer le chœur.

Les enseignements d'un scandale

17 Ces documents nous fournissent une multitude d'informations sur l'environnement personnel et institutionnel des deux musiciennes. Tout d'abord, la lettre d'Andrianna Ferrarese nous indique que les jeunes femmes s'étaient rendues à plusieurs reprises chez un personnage important de Venise pour y faire de la musique. En théorie, il leur était pourtant formellement interdit de jouer hors de leur institution. Même lorsqu'il y avait concert dans la chapelle de l'ospedale, les musiciennes jouaient derrière des grilles, dérobées au regard des auditeurs (cf. ill. 1). Rousseau en fit l'amère expérience, qui écrit dans ses Confessions ${ }^{10}$ :

Une musique à mon gré bien supérieure à celle des opéras, et qui n'a pas sa semblable en Italie ni dans le reste du monde, est celle des scuole. (...) Tous les 
dimanches, à l'église de chacune de ses scuole, on a durant les vêpres des motets à grand chœur et en grand orchestre, composés et dirigés par les plus grands maitres de l'Italie, exécutés dans des tribunes grillées uniquement par des filles dont la plus vieille n'a pas vingt ans. Je n'ai l'idée de rien d'aussi voluptueux, d'aussi touchant que cette musique : les richesses de l'art, le goût exquis des chants, la beauté des voix, la justesse de l'exécution, tout dans ces délicieux concerts concourt à produire une impression qui n'est assurément pas du bon costume, mais dont je doute qu'aucun cœur d'homme soit à l'abri. Jamais Carrio ni moi ne manquions ces vêpres aux Mendicanti, et nous n'étions pas les seuls. L'église était toujours pleine d'amateurs ; les acteurs mêmes de l'Opéra venaient se former au vrai goût du chant sur ces excellents modèles. Ce qui me désolait était ces maudites grilles, qui ne laissaient passer que ces sons, et me cachaient les anges de beauté dont ils étaient dignes.

Les concerts privés, dans les murs des ospedali, restaient exceptionnels et étaient réservés aux hôtes de marque ${ }^{11}$. Des exceptions étaient parfois faites, lorsque la République de Venise souhaitait honorer ses visiteurs illustres en leur offrant un concert. En 1782, par exemple, les meilleures solistes des quatre ospedali interprétèrent une cantate intitulée Telemaco nell'isola di Ogigia en l'honneur du «Comte et de la comtesse du Nord », c'est-à-dire du fils de la tsarine Catherine II, Paul II Petrovic et de son épouse Maria Teodorowna ${ }^{12}$, venus incognito.

Il est très rare de trouver des documents prouvant que l'interdiction de jouer en public n'était pas respectée. La lettre de la Ferrarese offre donc un témoignage d'autant plus précieux qu'il est quasiment unique.

Ce dossier fournit également à l'historien des informations précises sur les liens qu'entretenaient les filles du chœur avec les familles patriciennes de Venise. Les lettres de la Ferrarese à la procuratrice Giovanelli indiquent que la chanteuse avait une protectrice dans une famille influente de Venise, celle d'un procurateur de Saint$\operatorname{Marc}^{13}$. De fait, toutes les maestre des ospedali entretenaient des liens étroits avec une ou plusieurs familles patriciennes. Les bienfaiteurs profitaient fréquemment du lien de leur épouse avec un ospedale pour se faire élire gouverneur de celui-ci, poste qui menait parfois jusqu'à la dogature. Les bienfaiteurs des ospedali pouvaient également se vanter d'accomplir leur devoir religieux en prenant comme protégée une pauvre orpheline. En outre, ils profitaient des talents musicaux de celle-ci lors de soirées privées dans leurs palais, ou pour de plus longues périodes lorsqu'ils obtenaient l'autorisation d'emmener pour un mois leurs protégées in Villa, dans leurs villas de Terre Ferme. Les archives n'ont pas encore permis de prouver que les concerts organisés par les patriciens dans leurs villas avaient pour protagonistes les «filles du chœur ", mais il est peu probable que seules la générosité et la volonté de faire respirer le grand air à leurs protégées expliquent la constance remarquable avec laquelle ils sollicitaient les gouverneurs des ospedali pour obtenir l'autorisation d'emmener pour un mois leurs meilleurs musiciennes hors de Venise.

21 Les orphelines tiraient également avantage à avoir pour bienfaiteur des personnages haut placés dans le patriciat vénitien. En effet, le règlement très strict des ospedali impliquait que chaque pensionnaire se fit accompagner lorsqu'elle quittait l'ospedale pour plus d'une journée. Les raisons les plus fréquemment invoquées étaient le besoin de "grand air » et l'accomplissement des devoirs religieux. Lorsque les pensionnaires n'avaient pas de famille à Venise, elles pouvaient se mettre sous la protection d'un gouverneur de l'ospedale ou d'un membre de sa famille. Cette protection leur permettait d'obtenir des autorisations de sortie et leur offrait parfois des avantages substantiels. 
Lorsque son protecteur décédait, il n'était pas rare que la musicienne reçoive par testament un petit pécule, destiné à gonfler la dot octroyée par l'ospedale. Les relations entre bienfaiteur et protégée pouvaient prendre la forme de sentiments paternels ou amicaux très forts. Dans le cas d'Andrianna Ferrarese et de la procuratrice Giovanelli, les liens d'amitié semblaient réels, mais restaient marqués par la distance due au rang de la bienfaitrice. Si Andrianna Ferrarese l'appela à l'aide lors de sa mésaventure, alors qu'elle n'écrivit ni à son père, ni à un proche, elle ne l'appela jamais par son prénom et marqua sa déférence en utilisant toujours son titre («Procuratrice»). Néanmoins, une lettre plus ancienne de la Ferrarese, également présente dans le dossier, indique que sa bienfaitrice était suffisamment proche d'elle pour lui avoir prêté un manteau, ce qui implique une certaine confiance et un certain degré d'intimité entre les deux femmes.

Ce dossier nous renseigne enfin sur les aspirations des filles du chœur à une vie meilleure. Bien que les ospedali aient représenté pour elles une chance extraordinaire d'ascension sociale, elles ne semblent pas s'être toujours épanouies dans cet univers rigide. C'étaient, dans leur grande majorité, des jeunes filles influençables qui étaient entrées très jeunes dans l'institution et n'avaient presque rien connu de la vie à l'extérieur de ses murs. Elles rêvaient d'une vie meilleure, moins contrainte, sans voir les dangers qui les attendaient à l'extérieur. Andrianna Ferrarese n'était toutefois pas aussi ignorante qu'il y paraît à première vue. En effet, lorsqu'Agostino dal Bene lui suggèra de tenter la fortune à l'opéra, elle lui répondit qu'elle "irait volontiers à condition de trouver à se marier car si elle y allait ainsi, sans mari, cela ferait mauvais effet». Ce à quoi le consul de Rome lui répondit qu'elle était folle, et que «les femmes de théâtre n'ont pas de préjugés ", que " celle qui le veut peut se divertir, même si elle n'est pas mariée $»^{14}$. Ce à quoi la Ferrarese ne trouva rien à répondre.

Les musiciennes ont également conscience de leur talent, et pensent parfois qu'il pourrait s'épanouir à l'opéra. Dans sa "confession », Bianca Sacchetti écrivait qu'elle s'était laissée griser («échauffer la tête » est le terme qu'elle emploie) par l'idée du succès qu'elle pourrait obtenir sur les planches ${ }^{15}$. Andrianna Ferrarese écrivit quant à elle qu'Agostino dal Bene «ne manquait jamais de nous mettre en tête de fuir toutes les deux l'établissement, car avec notre talent pour le chant et la musique [instrumentale], nous pourrions faire une immense fortune en parcourant le monde et en faisant des académies [c.-à-d. des concerts privés]. Dans les premiers temps, nous pensions que ses discours n'étaient que des plaisanteries, et nous n'y avions pas prêté attention, mais comme il insistait toujours davantage, avec son grand pouvoir de persuasion, et devant son fils, il nous a tellement embobinées que nous avons pris le parti de faire ce qu'il disait ${ }^{16}$. Le consul de Rome était certainement beau parleur, il n'en reste pas moins que les musiciennes devaient se rendre compte de leur talent, surtout lorsque à la fin du XVIII ${ }^{e}$ siècle elles étaient sollicitées par la République pour honorer les personnages importants de passage à Venise.

Pourquoi prendre la fuite?

Pour pouvoir quitter l'ospedale, une musicienne du chœur devait impérativement remplir quatre conditions : avoir servi le chœur pendant au moins dix ans, avoir formé au moins deux musiciennes plus jeunes, obtenir des gouverneurs de l'établissement l'autorisation de se marier ou d'entrer au couvent et s'engager à ne plus jamais jouer ou chanter en public. Celles qui prenaient le voile obtenaient alors une dot de 200 ducats, celles qui se mariaient, une dot de 164 ducats et 12 grossi. Ni Andrianna Ferrarese ni Bianca Sacchetti ne remplissaient les conditions requises. On sait qu'elles ne 
souhaitaient pas cesser leur activité musicale, la présence de partitions dans le maigre trousseau qu'emporte Andrianna Ferrarese dans sa fuite le prouve. Bien loin de vouloir renoncer à leur talent, les deux musiciennes quittaient une institution qui empêchait celui-ci de s'épanouir à sa juste mesure. Elles pensaient pouvoir tenter leur chance à l'opéra, comme le leur avait conseillé le consul de Rome, et souhaitaient simplement changer de répertoire.

Toutefois, cela n'est probablement pas la raison principale de leur fugue, car les archives offrent plusieurs exemples d'anciennes « filles du chœur » ayant réussi à faire carrière sur les planches malgré l'interdiction formelle qui leur avait été donnée. Faustina Bordoni, ancienne chanteuse de la Pietà et épouse du compositeur Johann Adolf Hasse, se produisit ainsi dans toute l'Europe et devint même la rivale de la diva Francesca Cuzzoni au King's Theater de Londres dans la première moitié du XVIII siècle. Maddalena Lombardini-Sirmen, violoniste virtuose et ancienne élève de Tartini, quitta les Mendicanti pour épouser Lodovico Sirmen et fit la fortune de son mari en jouant ses œuvres dans toutes les cours d'Europe. Elle était également l'une des rares compositrices à avoir été formée dans les ospedali de Venise.

La véritable raison de la fugue de Bianca Sacchetti et Andrianna Ferrarese fut sans doute l'impossibilité d'obtenir l'autorisation de se marier, donc de quitter l'ospedale. En effet, Andrianna Ferrarese servait le chœur depuis quatre ans seulement en 1783, et Bianca Sacchetti depuis moins longtemps encore. Elles n'auraient jamais obtenu l'autorisation de se marier sans avoir rempli leur «engagement décennal» et sans avoir formé les quatre musiciennes censées leur succéder comme solistes. En outre, Andrianna Ferrarese était alors l'une des chanteuses principales des Mendicanti, comme le prouve sa présence sur tous les livrets depuis 1780, et Bianca Sacchetti était en passe de le devenir (elle apparaît à son tour sur tous les livrets à partir de 1783). On sait qu'il était plus facile pour une musicienne médiocre d'obtenir l'autorisation de se marier, les gouverneurs étant très réticents à laisser partir leurs meilleurs éléments, qui leur garantissaient un bon niveau d'exécution musicale, donc des rentrées d'argent régulières. Maddalena Lombardini-Sirmen, considérée comme la meilleure violoniste du chœur, avait ainsi dû demander à deux reprises l'autorisation de se marier avant que celle-ci lui soit accordée par les gouverneurs des Mendicanti ${ }^{17}$.

Il est un autre élément, plus difficile à évaluer, qui aurait pu inciter les gouverneurs à refuser à Andrianna Ferrarese l'autorisation de se marier. Outre le fait que l'autorisation du père du futur marié était indispensable, et aurait manqué à Luigi dal Bene, il est probable que les gouverneurs auraient considéré que ce dernier n'était pas un candidat sérieux au mariage et qu'ils auraient interdit à Andrianna Ferrarese de quitter l'ospedale pour lui. En effet, avant d'accorder leur autorisation, les gouverneurs menaient une enquête destinée à vérifier que le prétendant serait capable d'assumer financièrement la charge du ménage et qu'il était de bonnes mœurs. L'attitude de Luigi dal Bene vis-à-vis d'Andrianna Ferrarese après « l'affaire » indique que ses intentions à son égard étaient loin d'être louables. Après lui avoir promis - par écrit - le mariage et avoir "fait avec elle comme mari et femme ", selon la formule que l'on trouve dans les archives, il n'avait pas hésité à s'enfuir hors de la République de Venise et avait refusé, malgré toutes ses protestations d'amour, d'y revenir pour épouser la musicienne. Le dossier conserve plusieurs lettres des deux amants, dans lesquelles on peut lire la détresse d'Andrianna Ferrarese, déshonorée et incapable dorénavant de trouver un mari. Elle mit longtemps à réaliser que Luigi dal Bene avait abusé de sa crédulité. Ses 
lettres ne sont pas datées, mais on peut suivre l'évolution des sentiments de la musicienne au fil de ses courriers : les premières, adressées à la procuratrice Giovanelli, lui demandent de l'aider à obtenir les « formalités ecclésiastiques » qui valideraient son «mariage ». La dernière, adressée aux Provéditeurs aux hôpitaux et aux pieux établissements un an et demi après l'affaire, explique comment elle a été «séduite et trompée » par Luigi dal Bene et son père, qui lui affirme que «son fils ne serait jamais [son] mari car cela risquerait de lui faire perdre le consulat $»^{18}$.

Epilogue

L'attitude du consul de Rome dans cette affaire est plus importante qu'il n'y paraît à première vue. En effet, c'est lui qui incita les jeunes femmes à fuir l'ospedale pour tenter leur chance à l'opéra. En outre, il fut également celui qui rendit l'affaire publique, en allant lui-même dénoncer son fils aux gouverneurs de l'ospedale, afin d'éviter que le scandale de la fugue des musiciennes ne rejaillisse sur sa famille. La lettre adressée par les présidents des Mendicanti au Conseil des Dix relate l'événement, en insistant sur l'inquiétude manifestée par le père de Luigi dal Bene et sur sa volonté de retrouver son fils et ses « prisonnières » avant que l'irréparable ait été commis. Soi-disant honteux du comportement de Luigi, il promettait même de financer les recherches, pour peu que celles-ci fussent fructueuses ${ }^{19}$. Son comportement n'éveilla pas l'attention du président de l'ospedale, car Agostin dal Bene donnait l'image d'un bon père de famille, soucieux d'éviter le scandale à sa famille et à l'institution à laquelle appartenaient les musiciennes. Sans les lettres de Bianca Sacchetti et d'Andrianna Ferrarese, Agostin dal Bene n'aurait jamais été inquiété et son rôle véritable dans cette affaire serait resté inconnu.

La lettre d'Andrianna Ferrarese évoque les propositions malhonnêtes que lui avait faites le père de Luigi, et sa version est corroborée par la confession de Bianca Sacchetti, qui écrivit: "Agostino dal Bene, qui m'invitait souvent avec mon amie [Andrianna Ferrarese] à déjeuner chez lui, m'a dit à plusieurs reprises que j'étais folle de rester dans cet endroit à sacrifier mon talent (sono pazza a stare in questo loco a sacrificare le mie virto), et que je gagnerais des fortunes, et que lui-même me donnerait trois cents sequins pour aller de par le monde $»^{20}$. Les présidents de l'ospedale firent mener l'enquête, et l'implication d'Agostino dal Bene dans la fugue de son fils et des musiciennes put être prouvée. La promesse de mariage de Luigi dal Bene fut reconnue valide, et son père fut condamné à verser 485 ducats à Andrianna Ferrarese (soit le triple de la dot offerte habituellement par l'ospedale). Si jamais Luigi dal Bene avait décidé de tenir sa promesse, son père n'aurait pas à verser la somme susdite, celle-ci étant uniquement destinée à compenser le tort irréparable subi par la Ferrarese, qui risquait de compromettre fortement ses chances de mariage. Mieux dotée, elle avait une chance supplémentaire de trouver un mari qui feindrait d'ignorer qu'elle n'était plus tout à fait jeune fille.

Une étude de la rhétorique employée par les différents acteurs de cette affaire nous indique que seule Andrianna Ferrarese était encore dupe de Luigi dal Bene. Les autres protagonistes - les présidents de l'ospedale, l'envoyé du Grand Conseil Leonardo Capitanacchi - emploient tous le terme d' "enlèvement », ce qui affirme clairement le statut de victime de la musicienne. Même après que les jeunes femmes avaient été ramenées à l'ospedale, et que la Ferrarese protestait de sa volonté de fuir avec Luigi dal Bene, Leonardo Capitanacchi évoquait simplement l'« erreur » commise par la jeune femme, en indiquant que la faute en incombait à Luigi dal Bene, beau parleur qui avait 
su la convaincre de fuir avec lui. Le père de la jeune femme lui-même, prévenu par Leonardo Capitanacchi, ne jeta pas l'opprobre sur sa fille, se contentant de parler de "funeste nouvelle». Il ne la renia pas et accepta de recevoir sa fille chez lui une fois qu'elle eût été expulsée de l'ospedale.

31 Car malgré l'indulgence dont semblent faire preuve les présidents des Mendicanti et malgré la compensation financière obtenue, qui prouve la culpabilité de Luigi dal Bene, Andrianna Ferrarese ne pouvait pas «demeurer dans un lieu destiné aux vierges, qualité qu'elle [avait] notoirement perdue », écrivit Leonardo Capitanacchi au père de la jeune femme. Elle fut donc expulsée des Mendicanti, et renvoyée chez son père. Si ce châtiment peut sembler sévère, il s'explique par l'importance primordiale de la réputation dans la Venise du XVIII ${ }^{\mathrm{e}}$ siècle $^{21}$. Les Mendicanti, comme les autres ospedali, accordait une grande importance à la réputation sans tâche de ses pensionnaires, garante de la survie financière de l'institution - qui reposait, rappelons-le, sur les dons et les legs de généreux bienfaiteurs. En outre, la somme importante que fut condamné à verser Agostino dal Bene prouve s'il en était besoin que les présidents des Mendicanti avaient tenté d'assurer l'avenir de la musicienne, même lorsque celle-ci eût quitté l'ospedale.

32 La situation de Bianca Sacchetti est un peu différente. En effet, celle-ci fit amende honorable dès son retour à l'ospedale, elle écrivit qu'elle avait été entrânée dans cette affaire par Luigi dal Bene, son père, et Andrianna Ferrarese. Elle dit avoir été " séduite " par la fortune qu'ils lui faisaient miroiter et les perspectives d'avenir qui semblaient s'ouvrir à elle hors des murs des Mendicanti. Les présidents de l'ospedale furent d'autant plus enclins à la croire qu'elle était alors l'une des meilleures chanteuses du chœur, et qu'elle venait de finir sa formation. La renvoyer elle aussi serait revenu à perdre d'un seul coup deux des meilleures chanteuses de l'institution. En outre, la sage-femme confirma les dires de la jeune fille, à savoir qu'elle était encore pucelle. Rien n'obligeait donc les autorités à la renvoyer de l'ospedale, et elle fut effectivement réintégrée rapidement au sein des filles du chœur, où elle commença la même année une carrière de soliste qu'elle poursuivit pendant près de quinze ans.

Cette affaire eut des conséquences très différentes pour les deux musiciennes. Bianca Sacchetti resta toute sa vie aux Mendicanti, et apparaît sur la majorité des livrets de motets et d'oratorio écrits pour l'ospedale entre 1783 et 1797. Chanteuse, harpiste, flûtiste et organiste, elle occupa le poste de prieure - c'est-à-dire de responsable des filles du chœur, sommet de la hiérarchie pour une musicienne - jusqu'en 1805, après même que l'ospedale eût cessé toute activité musicale. Ce fut pour elle, semble-t-il, que Joseph Haydn composa la cantate Ariadne ${ }^{22}$.Ce fut également elle qui, à la fin du XVIII ${ }^{e}$ siècle, refusa un oratorio composé pour elle par Niccolò Antonio Zingarelli parce qu'elle trouvait que le style de l'œuvre n'était pas approprié au lieu sacré dans lequel il devait être chanté... montrant ainsi qu'elle avait définitivement troqué le goût de l'aventure pour la dévotion ${ }^{23}$.

34 Andrianna Ferrarese, quant à elle, réapparut en janvier 1785, au King's Theatre de Londres, où elle chanta le Demetrio de Luigi Cherubini. William Parke écrit que bien qu'elle eût été " très applaudie ", elle " avait une voix douce et chantait avec goût, mais n'était pas faite pour briller comme une prima donna $»^{24}$. Ce reproche la poursuivit tout au long de sa nouvelle carrière de cantatrice : on admira sa voix, et on regretta que son jeu d'actrice ne fût pas à la hauteur. Il est vrai que les musiciennes des ospedali étaient uniquement destinées à être entendues, non à être vues. Elles ne jouaient jamais 
aucune composition, pas même les oratorios pourtant de nature à être représentés sur une scène. Toutefois, il semblerait que cette lacune n'ait pas été rédhibitoire pour la Ferrarese, qui fit une brillante carrière sur les planches. Devenue la maîtresse de Da Ponte, elle chanta trois saisons à Vienne, entre 1788 et 1791. En août 1789, Mozart composa pour elle une aria («Un moto di gioia » K 579) et un rondo ( $\mathrm{Al}$ desio di chi t'adora » K 577) pour la reprise des Noces de Figaro. C'est également à Mozart qu'elle dut son rôle le plus célèbre, celui de Fiordiligi dans Così fan tutte, qu'elle créa le 26 janvier 1790.

Qu'elles aient fait carrière comme prima donna sur les scènes de théâtre européennes ou prieure dans un ospedale vénitien, les deux musiciennes surent donc exploiter l'enseignement musical de haut niveau qu'elles avaient reçu. L'une dans le domaine sacré, l'autre dans le domaine profane, elles restèrent les "servantes de la musique " évoquées par le père Vincenzo Coronelli au début du siècle (cf. ill. 2).

\section{BIBLIOGRAPHIE}

Sources

Venise, Archivio di Stato (abr. : I-Vas), Provveditori sopra Ospedali e Luoghi pii (abr. : Prov. sopra Osp. Lp.), B 80.

Venise, Istituto di Ricovero e di Educazione (abr. : I-Vire), Men. B 7.

Bibliographie

ARNOLD Elsie et BALDAUF-BERDES Jane, 2002, Maddalena Lombardini-Sirmen, Eighteenth-century

composer, Violonist and businesswoman, Lanham (Mld) et Londres, The Scarecrow Press.

BALDAUF-BERDES Jane, 1993, Women musicians of Venice : musical foundations (1525-1855), Oxford, Clarendon Press.

BOERIO Giuseppe, 1856, Dizionario del dialetto veneziano, Venise, Cecchini.

BURNEY Charles, 1776, Voyage musical dans l'Europe des Lumières, trad. de l'angl., éd. Michel Noiray, Paris, Flammarion, 1992.

CAFFI Francesco, 1835, Lettera ad E. Cicogna intorno alla vita ed al comporre di Antonio Lotti, Maestro di Cappella di San Marco di Venezia, Venise, s.n.

cowAN Alexander, 2006, "Looking in and looking out »: observation, gossip and street culture in early modern Venice, n. p.

LANE Frederic C., 1985, Venise : une république maritime, trad. de l'angl., réed., Paris, Flammarion. LEWY GIDWITZ Patricia et RICE John A., 2001, « Andrianna Ferrarese », in Stanley Sadie (éd.), New Grove dictionary of music and musicians, Londres, Macmillan.

PARKE William T., 1830, Musical memoirs, Londres, H. Colburn and R. Bentley. 
PULLAN Brian, 1989, « La nuova filantropia nella Venezia cinquecentesca », in Bernard Aikema (dir.), Nel regno dei poveri : arte e storia dei grandi ospedali veneziani in età moderna (1497-1797), Venise, Arsenale.

ROUSSEAU Jean-Jacques, 1782, Confessions, nouv. éd., Paris, Flammarion, 2002.

\section{NOTES}

1. Trad. : « Fuite de Ferrarese et Sacchetti ».

2. «Puta : pulcella; donzella; fanciulla; femmina nubile, giovane da marito» (Boerio 1856 : 541). Ce terme, synonyme de vierge pour les Vénitiens, désigne fréquemment les « filles du chœur » des ospedali de Venise.

3. Pullan $1989: 20$.

4. Maître de chœur aux Mendicanti entre 1740 et 1751, Baldassare Galuppi composa la partition de L'arcadia in Brenta en 1749, Il mondo della luna, Arcifanfano re dei matti, Il paese della Cuccagna et Il mondo alla roversa en 1750.

5.I-Vire, Men. B 7, $n^{\circ}$ 7724, 26 septembre 1774.

6. Abraham sacrificium, Actio sacra pro virginibus choristis S. Lazari Mendicantium modulis ornata a celeberrimo musices magistro Francisco Bianchi cremonensi Accademico Philarmonico Bononice, Venise, "ex typographia Petri Valvasensis", 1783, 16 p.

7. De Morte Sisarae Chananoerum ducis, Actio sacra pro virginibus choristis Sancti Lazari Mendicantium. Modos fecit D. Jo. Jacobus Avanzini cremensis recurrente dominica Pentecostes anni 1780 , Venise, s.n., $20 \mathrm{p}$.

8. Burney $1776: 130$.

9. I-Vas, Provv. sopra Osp. Lp., B 80, « Ferrarese e Sacchetti fuggite », s.d.

10. Rousseau 1782 : VII, 52-53. Rousseau fait ici une confusion en appelant scuole les ospedali. Cette inexactitude lexicale provient probablement du fait qu'il traduit littéralement le terme " école [de musique] » par son équivalent italien, scuola, ignorant probablement le sens particulier qu'avait ce terme à Venise, où il qualifie une confrérie de pénitents.

11. Jean-Jacques Rousseau eut cet honneur aux Mendicanti, et fut fort déçu de réaliser que les musiciennes n'étaient pas les « anges de beauté » auxquels il s'attendait : « En entrant dans le salon qui renfermait ces beautés si convoitées, je sentis un frémissement d'amour que je n'avais jamais éprouvé. M. Leblond me présenta l'une après l'autre ces chanteuses célèbres, dont la voix et le nom étaient tout ce qui m'était connu. Venez, Sophie... Elle était horrible. Venez, Cattina... Elle était borgne. Venez, Bettina... La petite vérole l'avait défigurée. Presque pas une n'était sans quelque notable défaut. Le bourreau riait de ma cruelle surprise. Deux ou trois cependant me parurent passables : elles ne chantaient que dans les chœurs. J'étais désolé. Durant le goûter, on les agaça ; elles s'égayèrent. La laideur n'exclut pas les grâces ; je leur en trouvai. Je me disais : « On ne chante pas ainsi sans âme ; elles en ont ». Enfin ma façon de voir changea si bien, que je sortis presque amoureux de tous ces laiderons. J'osais à peine retourner à leurs vêpres. J'eus de quoi me rassurer. Je continuai de trouver leurs chants délicieux, et leurs voix fardaient si bien leurs visages, que tant qu'elles chantaient je m'obstinais, en dépit de mes yeux, à les trouver belles » (Rousseau 1782 : VII, 52-53). 12. Telemaco nell'isola di Ogigia, cantata a sei voci, Venise, s.n., 1782.

13. La fonction de procurateur de Saint-Marc, élective, était conférée à vie. Lorsqu'elle avait été créée, au XII ̀̀ siècle, un salaire substantiel y avait été attaché, mais à l'époque 
moderne, cette fonction n'était plus rémunérée. Néanmoins, le titre de procurateur de Saint-Marc restait fort prisé pour les honneurs qui lui étaient attachés (Lane 1985 :

150).

14. I-Vas, Provv. sopra Osp. Lp., B 80, « Ferrarese e Sacchetti fuggite », s.d.

15. Ibidem.

16. Ibidem.

17. Sur la question du mariage de Maddalena Lombardini et les réticences des gouverneurs, voir Baldauf-Berdes et Arnold 2002 : 35-43.

18. I-Vas, Provv. sopra Osp. Lp., B 80, « Ferrarese e Sacchetti fuggite », s.d.

19. I-Vas, Provv. sopra Osp. Lp., B 80, « Ferrarese e Sacchetti fuggite », 13 janvier 1783 (n.st.).

20. I-Vas, Provv. sopra Osp. Lp., B 80, « Ferrarese e Sacchetti fuggite », s.d.

21. Sur ce sujet, voir Cowan 2006.

22. Rochlitz, « Korrespondenz », col. 336, cité par Baldauf-Berdes 1993 : 123-124.

23. Caffi 1835.

24. Parke $1830: 1,49$.

\section{RÉSUMÉS}

En janvier 1783, deux musiciennes, Bianca Sacchetti et Andrianna Ferrarese, s'enfuirent de l'ospedale des Mendicanti, à Venise, pour tenter leur chance sur les scènes de théâtre. Formées dans l'une des quatre institutions vénitiennes destinées à recueillir les orphelins de Venise, elles étaient devenues des musiciennes hors pair, chantaient chaque jour dans la chapelle de l'ospedale et jouaient de plusieurs instruments. Le dossier consacré à leur fugue, qui se trouve toujours à l'Archivio di Stato de Venise, nous donne les circonstances et les raisons de leur fugue, mais fournit également des informations précieuses et uniques sur les aspirations des « filles du choeur " à une vie meilleure. Des documents inédits illustrent les relations d'amitié qui pouvaient exister entre deux jeunes femmes recueillies par charité et suffisamment talentueuses pour avoir réussi à devenir deux des meilleures musiciennes de Venise. Retrouvées par les autorités, elles poursuivirent d'ailleurs une brillante carrière, l'une à l'opéra, l'autre au sein de l'ospedale, la Ferrarese créant même l'un des rôles du Così fan tutte de Mozart.

In January 1783, two women musicians, Bianca Sacchetti and Andrianna Ferrarese, fled from the ospedale of the Mendicanti, in Venice, to try their luck on the opera stage. They had been trained in one of the four Venetian institutions that took in the orphans of the city, and they had become outstanding musicians. They could sing and play several instruments. The file related to the story is still lying in the Archivio di Stato in Venice, and it offers precious information about the circumstances and the reasons for their escape. Reading it, we learn much about the desire of the "choir girls" for a better life. Unpublished documents illustrate the friendship existing between these two orphaned girls who were talented enough to became two of the best women musicians of Venice. When they were found by the authorities, they were able to pursue their brilliant career, at the opera house or in the church; the Ferrarese even created one of the roles in Mozart's Così fan tutte. 
INDEX

Index chronologique : XVIIIe siècle

Keywords : Venice, women musicians, XVIIIth

Mots-clés : Ferrarese, musiciennes, ospedale dei Mendicanti, Sacchetti

Index géographique: Venise

\section{AUTEUR}

\section{CAROLINE GIRON-PANEL}

Caroline GIRON-PANEL, archiviste paléographe, est conservateur des bibliothèques, en formation à l'Enssib. Après une thèse de l'Ecole des Chartes (2004) consacrée à « La vie musicale à l'ospedale di San Lazzaro e dei Mendicanti, à Venise (1600-1797) », elle prépare actuellement une thèse de doctorat intitulée «A l'origine des conservatoires : le modèle des ospedali vénitiens (XVI ${ }^{\text {-XVIII }}{ }^{\text {è }}$ siècles) ». Elle a également travaillé au répertoire des partitions manuscrites des œuvres de Baldassare Galuppi (1706-1785) à la Bibliothèque nationale de France. Elle a publié : « Une collection perdue : les instruments de l'ospedale des Mendicanti, à Venise ", dans Musique-ImagesInstruments, $\mathrm{n}^{\circ} 8,2006$, p. 28-47 et plusieurs autres articles sont à paraître. 